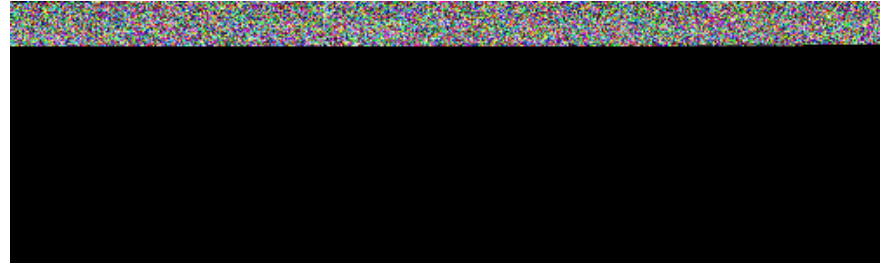

This information is current as of April 26, 2023.

\title{
FDG-PET/CT or MRI for the Diagnosis of Primary Progressive
}

\section{Aphasia?}

J.A. Matias-Guiu, M.N. Cabrera-Martín, J. Matías-Guiu and J.L. Carreras

AJNR Am J Neuroradiol 2017, 38 (9) E63

doi: https://doi.org/10.3174/ajnr.A5255

http://www.ajnr.org/content/38/9/E63 


\section{FDG-PET/CT or MRI for the Diagnosis of Primary Progressive Aphasia?}

W e have read with interest the article by Sajjadi et al $^{1}$ concerning the use of MR imaging for the diagnosis of primary progressive aphasia (PPA). The authors studied the sensitivity and specificity of the visual analysis of MR imaging for the diagnosis of each PPA variant. They found that accuracy values were appropriate for the semantic variant, but somewhat disappointing for logopenic and nonfluent aphasia. Although specificity values were above $90 \%$ for the 3 variants, sensitivity for nonfluent and logopenic PPA was $21 \%$ and $49 \%$, respectively. This issue is relevant because a biomarker showing focal neurodegeneration (eg, MR imaging) is included in the current diagnostic criteria and clinical diagnosis of PPA variants may be difficult. ${ }^{2}$

We published a similar study 2 years ago, but with FDG-PET/ CT. ${ }^{3}$ In our study, FDG-PET images from a cohort of 33 patients with PPA and 11 controls were visually reviewed by 5 nuclear medicine physicians to evaluate the diagnostic accuracy of the technique and the interrater agreement. Another 5 raters also reviewed the maps using Statistical Parametric Mapping (SPM), comparing each patient individually with a healthy control group (statistical analysis). Interrater agreement was moderate for visual analysis (Fleiss $\kappa=0.568$ ) and substantial for statistical analysis $(\kappa=0.756-0.881)$. Sensitivity and specificity for the diagnosis of PPA (to discriminate it from healthy controls) was $87.8 \%$ and $89.9 \%$, respectively, in the visual analysis. Interrater agreement was high in semantic and logopenic variants (at least 4 of 5 raters agreed in $100 \%$ and $84 \%$ of cases, respectively), and it was lower in nonfluent aphasia (at least 4 of 5 raters agreed in only $20 \%$ of cases). Furthermore, using images statistically preprocessed by SPM improved the agreement among raters, especially in the nonfluent variant.

We reanalyzed the data of our previous study ${ }^{2}$ with the same method as that used by Sajjadi et al. ${ }^{1}$ We estimated the sensitivity and specificity for the diagnosis of each type of PPA. Mean sensitivity and specificity of expert raters using FDG-PET was, respectively, $65 \%$ and $98.5 \%$ for the nonfluent variant, $62.5 \%$ and $96.2 \%$ for the semantic variant, and $89.4 \%$ and $86 \%$ for the logopenic type. With statistical analysis, the mean sensitivity and specificity were $70 \%$ and $94.1 \%$ for nonfluent, $75 \%$ and $93.5 \%$ for semantic, and $82.1 \%$ and $88 \%$ for the logopenic variant.

Thus, our study revealed higher diagnostic accuracy, especially regarding sensitivity, for the diagnosis of PPA and its variants with FDG-PET than that reported by Sajjadi et $\mathrm{al}^{1}$ with MR imaging. This might support a better diagnostic performance of FDGPET/CT compared with MR imaging in the specific setting of diagnosing and classifying PPA. However, studies directly comparing FDG-PET/CT and MR imaging accuracy are necessary to clarify the superiority of one technique over the other ${ }^{4}$ or to evaluate a potential benefit of the combination of both techniques at an individual level. Furthermore, the assessment of statistical maps may reduce some of the limitations encountered when performing direct visual analysis of images.

Disclosures: Jorge Matias-Guiu-UNRELATED: Board Membership: Spanish Neurological Society, Comments: Editor-in-Chief of Neurologia, official journal of the Spanish Neurological Society; Employment: Hospital Clinico San Carlos, Universidad Complutense de Madrid, Comments: Professor of Neurology, Director of Neuroscience Institutes, Head of Neurology Department.

\section{REFERENCES}

1. Sajjadi SA, Sheikh-Bahaei N, Cross J, et al. Can MRI visual assessment differentiate the variants of primary-progressive aphasia? AJNR Am J Neuroradiol 2017 Mar 24. [Epub ahead of print] CrossRef Medline

2. Gorno-Tempini ML, Hillis AE, Weintraub S, et al. Classification of primary progressive aphasia and its variants. Neurology 2011;76: 1006-14 CrossRef Medline

3. Matías-Guiu JA, Cabrera-Martín MN, Pérez-Castejón MJ, et al. Visual and statistical analysis of ${ }^{18} \mathrm{~F}$-FDG PET in primary progressive aphaia. Eur J Nucl Med Mol Imaging 2015;42:916-27 CrossRef Medline

4. Panegyres PK, McCarthy M, Campbell A, et al. Correlative studies of structural and functional imaging in primary progressive aphasia. Am J Alzheimers Dis Other Demen 2008;23:184-91 CrossRef Medline

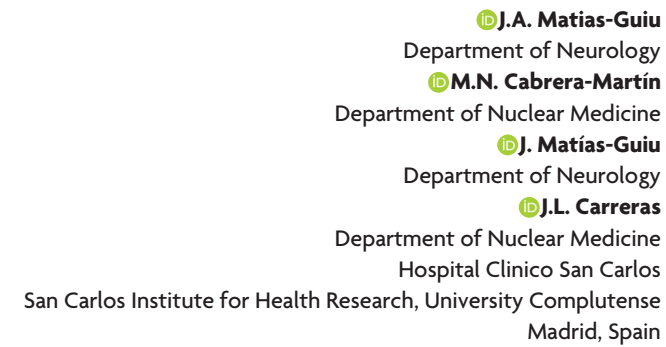

(D).A. Matias-Guiu Department of Neurology ent Nuclear Medicine (D). Matías-Guiu of Neurology tment of Nuclear Medicine Hospital Clinico San Carlos Madrid, Spain 